\title{
ARTICLES
}

\section{Identification of a Prosencephalic-Specific Enhancer of SALL1: Comparative Genomic Approach Using the Chick Embryo}

\author{
KOSUKE IZUMI, MICHIHIKO ARAMAKI, TOKUHIRO KIMURA, YOKO NAITO, TORU UDAKA, MASANORI UCHIKAWA, \\ HISATO KONDOH, HIDEKAZU SUZUKI, GINAM CHO, YASUNORI OKADA, TAKAO TAKAHASHI, \\ JEFFREY A. GOLDEN, AND KENJIRO KOSAKI
}

\begin{abstract}
Department of Pediatrics [K.I., M.A., Y.N., T.T., K.K.], Department of Pathology [T.K., Y.O.], Department of Internal Medicine [H.S.], Keio University School of Medicine, Tokyo 160-8582, Japan; Graduate School of Frontier Biosciences [M.U., H.K.], Osaka University, Suita 565-0871, Japan; Department of Pathology [G.C., J.A.G.], Children's Hospital of Philadelphia and the University of Pennsylvania School of Medicine, Philadelphia, PA 19104
\end{abstract}

\begin{abstract}
Comparative genomics is a promising approach for identifying regulatory elements governing the unique spatio-temporal expression patterns of morphogenetic genes. Conserved noncoding genomic sequences are candidate regulatory elements. Here we performed a survey for conserved noncoding elements (CNE) nested within the SALL1 gene; mutations in this gene result in the TownesBrocks syndrome. A comparison of the genomic sequence between humans and chicken revealed five CNE. Genomic fragments corresponding to each CNE were inserted into reporter cassettes consisting of $e G F P$ cDNA and a minimal promoter. These constructs were electroporated into chick embryos during gastrula, neurula, and pharyngula stages. Among the five CNE that were examined, one 443 bp CNE exhibited tissue-specific enhancer activity. At the neurula stage, the eGFP signal was visualized in the prosencephalon. At the pharyngula stage, the eGFP signal was confined within the anterior neural ridge, which represents one of the morphogenetic centers regulating the patterning of the anterior neural plate. This report identifies, for the first time, an enhancer element of SALL1. (Pediatr Res 61: 660-665, 2007)
\end{abstract}

A dvances in comparative genomics have begun predicting potential regulatory elements, because these essential sequences tend to be conserved through evolution $(1,2)$. Furthermore, whole-genome comparison studies among several species revealed that noncoding genomic sequences that are well conserved through evolution (CNE) are clustered in the vicinity of known transcription factors that play critical roles in embryogenesis (3). Hence, CNE represent reasonable candidates for potential regulatory elements.

Received September 6, 2006; accepted February 4, 2007.

Correspondence: Kenjiro Kosaki, M.D., Keio University School of Medicine, Department of Pediatrics, 35 Shinanomachi, Shinjuku-ku, Tokyo, 160-8582, Japan; e-mail: kkosaki@sc.itc.keio.ac.jp

This research was partly supported by a Grant-in-Aid for Exploratory Research from the Japanese Ministry of Education, Culture, Sports, Science and Technology. K.I. was partly supported by a research grant from the Center of Excellence (COE) program "Basic Study and Clinical Application of the Human Stem Cell Biology and Immunology."

This article contains online supplemental material at www.pedresearch.org.

DOI: $10.1203 / p d r .0 b 013 e 318053423 a$
In a whole-genome comparative study using pufferfish, Fugu rubripes, the transcription factor SALL1 was found to possess a relatively abundant number of CNE in proximity to the coding sequence (3). However, the in vivo function of these CNE has not been examined. Here, we attempted to perform a systematic functional survey for CNE belonging to the SALLl gene, an important developmental regulatory molecule. In the present study, we specifically studied the noncoding region embedded between the putative transcription start site and the stop codon. SALL1, a zinc-finger transcription factor located on 16q12.1 in human genome, has been identified as one of the four human homologues of the Drosophila region-specific homeotic gene spalt (sal) (4). Heterozygous mutations in human SALL1 genes cause TownesBrocks syndrome, characterized by anal defects, upper limb preaxial defects, first and second arch defects including the ears and jaw, kidney malformations, and occasionally mental retardation $(5,6)$. Concordant with the distribution of these malformations, SALL1 is expressed in a unique spatiotemporal pattern in various species. In mice, Sall1 is expressed in the limbs, ear, anus, heart, kidney, spinal cord and brain $(7,8)$. csall, the chicken ortholog of SALL1, is expressed in the heart, the pharynx, involuting mesoderm and the neural plate with subsequent strong expression in the neural tube of the early embryo (9). In later developmental stages, csall expression is also found in the tail bud and developing limb buds (10).

To identify the cis-acting regulatory elements for SALL1, we first performed a comparative genomic scan that yielded five CNE. We next took advantage of chick model system. As a vertebrate species, chick undergoes early embryogenesis that is remarkably similar to that of human in morphologic change as well as expression patterns of developmental transcription factors. Furthermore, the size of the chicken genome is only $40 \%$ of that of human genome without considerable differ-

Abbreviations: CNE, conserved noncoding element; HH, Hamburger-Hamilton; Shh, Sonic hedgehog 
ences in the number of the genes and this compact nature of chicken genome facilitates the identification of putative enhancer elements (1). Furthermore, electroporation to the chick embryo has simplified gene transfer, facilitating rapid genetically based studies (11). Using this system, we have identified one CNE that specifically directs $S A L L 1$ to the forebrain, one of the regions where SALL1 is expressed.

\section{MATERIALS AND METHODS}

Identification of CNE in the vicinity of SALL1. We performed a bioinformatic analysis using a human genome sequence of roughly $115 \mathrm{~kb}$ covering SALL1. The base position coordinates of the target region on chromosome 16 from 16pter were 49677829-49792653, as determined according to the May 2004 human reference sequence NCBI Build 35 assembled by UC Santa Cruz genome browser (http://genome.ucsc.edu/). The chicken genome sequence, a Gallus gallus chromosome 11 genomic contig (GenBank accession number: NW_060474.1) covering the chicken SALL1 ortholog, was obtained using the NCBI map viewer (http://www.ncbi.nlm.nih.gov/mapview/). These two sequences were compared to identify CNE using the Mulan website (http://mulan.dcode.org/) (12). In the present study, we defined CNE as stretches of base pairs at least 100 bases in length and with a similarity of at least $70 \%$ between the human and chicken. We named the chicken $\mathrm{CNE}$ as corresponding $\mathrm{CNE}$ sequences from the chicken genome and human $\mathrm{CNE}$ as corresponding CNE sequences from the human genome. Transcription factor binding sites were predicted using rVISTA2.0 software (http://rvista.dcode. org/) (13).

Construction of reporter eGFP expression cassettes. Genomic fragments corresponding to each CNE were prepared using PCR and inserted into reporter cassettes consisting of $e G F P$ cDNA and a minimal promoter. The PCR primers were designed for each CNE using the Primer 3 website (http://frodo.wi.mit.edu/cgi-bin/primer3/primer3_www.cgi). The primer sequences are listed in a supplemental Table $1 \bar{A}$ (material online at www. pedreasearch.org). The PCR products were treated with PWO DNA polymerase (Roche Molecular Biochemicals, Indianapolis, IN) and T4 polynucleotide kinase (Takara, Kyoto, Japan) to facilitate cloning. One of the CNE (CNE3) was divided into four blocks: subregion I (100 bp), subregion II (157 bp), subregion III (92 bp), and subregion IV (110 bp). Deletion constructs lacking each subregion were created using the Splicing by Overlap Extension PCR (SOEing PCR) technique (Fig. 1) (14). The region $5^{\prime}$ to the segment to be deleted and that $3^{\prime}$ to the segment were independently generated using PCR. These two PCR products were joined together using the SOE method and four kinds of PCR primers: an outer forward primer, an inner reverse primer, an

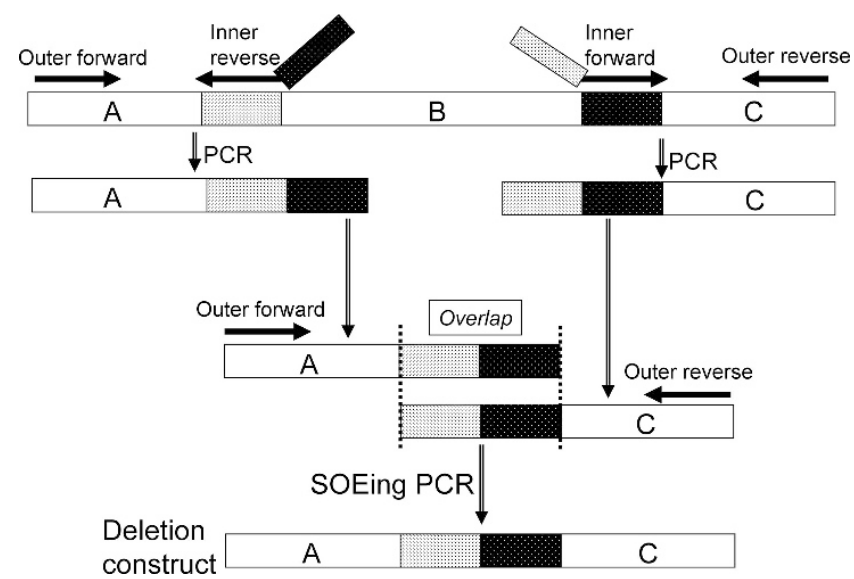

Figure 1. Schematic diagram of SOEing PCR. Two regions $(A, C)$ flanking the region to be deleted $(B)$ were fused by SOEing PCR. The fusion is mediated by an overlap of the two strands of PCR products that were created with the use of primers (outer forward primer, inner reverse primer, inner forward primer, outer reverse primer). $5^{\prime}$-region of inner reverse primer used in the amplification of region $A$ is complimentary to the segment of region $C$. 5 '-region of inner forward primer used in the amplification of region $C$ is complimentary to the segment of region $A$. A recombinant product is formed when this overlap is extended in a subsequent reaction, and this recombined product is amplified with outer primers. inner forward primer, and an outer reverse primer (supplemental Table $1 B$, material online at www.pedresearch.org). The $5^{\prime}$-half of the inner reverse primer was designed to be complementary to the inner forward primer, and the $5^{\prime}$-half of the inner forward primer was designed to be complementary to the inner reverse primer. These complementarities between the inner primers allowed them to overlap when the PCR products were mixed, denatured, and reannealed. Amplification of the overlapped molecules by the outer forward and outer reverse primers and DNA polymerase led to the extension of the overlap, thereby "splicing" the original PCR products. Using T4DNA ligase (Takara), PCR products were inserted into the polylinker SmaI site of ptkEGFP. The plasmid ptkEGFP was constructed by insertion of the Herpes simplex virus thymidine kinase promoter in the polylinker HindIII site of pCAT3-basic vector (Promega, Madison, WI), and by replacing the CAT gene with the EGFP gene (CLONTECH, Palo Alto, CA) (11). The plasmid was cleaved by SmaI and then treated with CIAP (Invitrogen, Carlsbad, CA) and ligated with the PCR products. The plasmids were transformed into competent DH5 $\alpha$ Escherichia coli (Toyobo, Osaka, Japan) using a standard protocol and plated on LB agar containing ampicillin $(100 \mu \mathrm{g} / \mathrm{mL})$ as a selective antibiotic. Colonies were selected and expanded in LB medium containing ampicillin $(100 \mu \mathrm{g} / \mathrm{mL})$ in a rotary shaker at $37^{\circ} \mathrm{C}$. The plasmids were harvested using the Endofree plasmid Maxi kit (QIAGEN, Valencia, CA) and dissolved in DNA solution. The sequences of the cloned CNE elements were confirmed using an $\mathrm{ABI}$ prism 3100 sequence analyzer. After CNE insertion, the plasmid construct was transfected to early chick embryos. The study protocols were approved by the Animal Care Committee of Keio University School of Medicine.

Ex ovo electroporation. The plasmid construct was transfected using in vivo-ex ovo electroporation as described previously $(1,11)$. Briefly, after $20-24 \mathrm{~h}$ in an incubator, HH stage 5 chick embryos (15) were harvested. The thick albumen was partially removed, a ring of filter paper was placed on the vitelline membrane, and the membrane was cut at the circumference of the filter ring. The embryo anchored to the filter ring through the vitelline membrane was transferred into warmed Hank's solution. The embryo was then placed, with the vitelline membrane side downwards, onto an electrode (CUY700P2E; NEPA GENE, Chiba, Japan). A DNA solution containing the reporter plasmid (2 $\mu \mathrm{g} / \mu \mathrm{L}$ ) and the marker plasmid [pDsRed1-N1 (CLONTECH), $1 \mu \mathrm{g} / \mu \mathrm{L}$ ] was injected between the blastoderm and the vitelline membrane using a glass capillary. Glass capillary needles were pulled using a Narishige PN-3 horizontal puller (Narishige Scientific Instrument Lab, Tokyo, Japan) from borosilicate glass capillary tubing (GD-1, $90 \mathrm{~mm}$ in length, $1 \mathrm{~mm}$ outside diameter $\times 0.6 \mathrm{~mm}$ inside diameter; Narishige Scientific Instrument Lab); the needles were then beveled to a diameter of $0.05 \mathrm{~mm}$.

An anodal electrode (CUY700P2L; NEPA GENE) was placed on the hypoblast side of the embryo. Electroporation was performed using an OVODYNE electroporator (Intracel, Herts, UK) with five pulses of 8-10 V for a duration of $50 \mathrm{~ms}$ and with intervals of $100 \mathrm{~ms}$. The embryo was incubated at $38^{\circ} \mathrm{C}$ and $100 \%$ humidity. The anatomical region of successful electroporation was confirmed by the co-electroporation of a DsRed vector carrying a universal promoter. eGFP or DsRed signals were observed under LED lights. For eGFP excitation, lights from 12 blue LEDs (peak wave length: $475 \mathrm{~nm}$ ) were intensified using a concave lens and were projected onto the embryonic specimen through a band-pass filter (BI0060; Asahi Spectra, Tokyo, Japan). Emissions from the eGFP fluorescence were observed through a long-pass filter (FF01-520/35-25; Bright Line, Semrock, Rochester, NY). For the excitation of DsRed, lights from 12 green LEDs (peak wave length: $520 \mathrm{~nm}$ ) were projected through a band-pass filter (FF01-562/40-25; Bright Line). Emissions from DsRed were observed through a long-pass filter (610LP; Thin Film Imaging Technologies, North Mankato, MN). The results were demonstrated as superimposed images of the eGFP image and a light, bright field image.

In ovo electroporation. After about $40 \mathrm{~h}$ of incubation (HH stage 10) chick embryos were electoroporated using a slight modification of the original method (16). For in ovo electroporation, an EP21 current amplifier (Intracel) was used with an OVODYNE electroporator (Intracel). An anode (CUY610P4; NEPA GENE) was placed beneath the embryo, and a cathode (CUY610P4; NEPA GENE) was placed onto the cephalic region. A DNA solution was injected into the neural tube and electroporated with five pulses of $26 \mathrm{~V}$ for a duration of 50 $\mathrm{ms}$ and at intervals of $100 \mathrm{~ms}$. After the electroporation, the embryos were incubated for another $24-48 \mathrm{~h}$. The anatomical region of successful electroporation was confirmed by the co-electroporation of a DsRed vector. eGFP/DsRed signals were observed as described above.

Histologic analysis using plastic-embedded sections. To preserve eGFP signals for histologic analysis, the electroporated embryos were embedded in plastic resin (Technovit 8100, Heraeus Kulzer) according to the manufacture's instructions. Briefly, the embryos were fixed in $4 \%$ paraformaldehyde, washed overnight in PBS containing $6.8 \%$ sucrose at $4{ }^{\circ} \mathrm{C}$, dehydrated in 100\% acetone, and embedded in Technovit 8100 using Histoform Q (Heraeus 
Kulzer ) as a mold. The polymerized resin was fixed onto a block (Histobloc, Heraeus Kulzer) with Technovit 3040 (Heraeus Kulzer), and sections (5- to 6 - $\mu$ m-thick) were cut using a microtome. eGFP fluorescence was observed using a laser scanning confocal microscope (FLUOVIEW FV300; Olympus, Tokyo, Japan). The results were visualized as superimposed eGFP and Nomarski images. Nomarski images were obtained using differential interference contrast microscopy, which is an optical microscopy illumination technique used to enhance contrast in unstained samples.

In situ hybridization analysis. We performed the in situ hybridization analysis using a previously described protocol (17). Chick EST clones corresponding to the chicken Sall1 (csall) sequence (GenBank accession number, NM_204707) were identified using the chick EST database available on the Biotechnology and Biologic Sciences Research Council's website (http://www.chick.umist.ac.uk/) (18). Among the 19 EST clones identified by a database search, ChEST81814, which originates from the cDNA of chicken stage 22 limbs, was used as a template for riboprobe synthesis in this study.

\section{RESULTS}

Identification of conserved noncoding elements. A comparison of the genomic sequences of humans and chicken revealed eight conserved elements within the SALL1 gene. However, three of the eight elements were identical to the coding region and untranslated region of $S A L L 1$; thus, five elements were identified as CNE (Fig. 2). The percentage similarities of the CNE ranged from $75.77 \%$ to $91.93 \%$, and the lengths of the CNE varied from 210 bp to $443 \mathrm{bp}$ (Table 1). Five CNE were examined using ex ovo electroporation, and only one CNE, a 443-bp CNE in intron 1, exhibited tissue-specific enhancer activity. We named the enhancer sequence in intron 1 as $\mathrm{CNE} 3$. The similarity between the human CNE3 genomic sequence (base position coordinates on chromosome 16 from 16pter according to the May 2004 human reference sequence NCBI Build 35: 4973449849734940) and chicken CNE3 sequence was $81.33 \%$. The actual chicken CNE3 sequence data, which was cloned into the plasmid vector and was actually injected, is presented in the supplemental data (material online at www.pedreasearch.org). The other four CNE did not show any enhancer activity in early-stage embryos.

Enhancer activity of CNE3 during the neurula stage. When the chicken CNE3 plasmid construct was electroporated into the embryo at the gastrula stage (HH stage 5), the eGFP signal was visualized in the prosencephalon at $\mathrm{HH}$ stages 8-12 (Fig. 3). The presence of a DsRed signal covering the

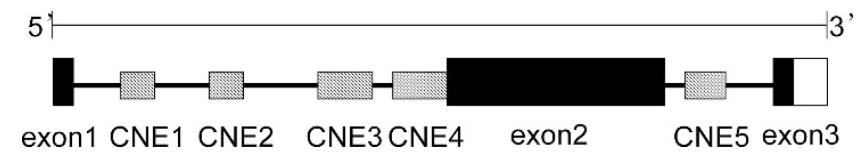

Figure 2. Genomic structure of $S A L L 1$. The closed boxes represent exons, the open box represents the UTR, and the shaded boxes represent CNEs.

Table 1. Characteristics of five CNE

\begin{tabular}{lcccc}
\hline & Genomic position & $\begin{array}{c}\text { Length } \\
\text { (human) }\end{array}$ & $\begin{array}{c}\text { Length } \\
\text { (chicken) }\end{array}$ & $\begin{array}{c}\text { Percentage } \\
\text { identity }\end{array}$ \\
\hline CNE1 & chr16:49737740-49738181 & $442 \mathrm{bp}$ & $444 \mathrm{bp}$ & $85.54 \%$ \\
CNE2 & chr16:49735568-49735977 & $410 \mathrm{bp}$ & $412 \mathrm{bp}$ & $91.93 \%$ \\
CNE3 & chr16:49734498-49734940 & $443 \mathrm{bp}$ & $442 \mathrm{bp}$ & $81.33 \%$ \\
CNE4 & chr16:49733832-49734041 & $210 \mathrm{bp}$ & $210 \mathrm{bp}$ & $75.77 \%$ \\
CNE5 & chr16:49729284-49729589 & $306 \mathrm{bp}$ & $304 \mathrm{bp}$ & $78.49 \%$ \\
\hline
\end{tabular}

(From Mulan software: http://mulan.dcode.org/. Searched on 2005/5/10. Request ID: m05100416557730.) entire chick embryos indicated that ex ovo electroporation was successful (Fig. 3A). In HH stage 8 embryos, eGFP fluorescence reflecting the enhancer activity, appeared in the neural tube around the anterior neural pore (before the completion of neural tube closure) (Fig. 3B). In HH stage 10 embryos, eGFP signals were present in the prosencephalon (Fig. $3 C$ ). In $\mathrm{HH}$ stage 12 embryos, eGFP signals were present throughout the entire prosencephalon (Fig. 3D). As the head-folding process progressed (HH stage 14), the eGFP signals became localized in the telencephalon (Fig. 3E). Insertion of the CNE3 sequence in the reverse direction did not alter eGFP expression at $\mathrm{HH}$ stages 8-12 (data not shown). Electroporation of the human CNE3 exerted a comparable enhancer activity at $\mathrm{HH}$ stages 9-10 (Fig. 4), but the eGFP signal became less prominent as development progressed. No eGFP signal was observed at HH stage 12 or later with human CNE3. Serial axial sections of HH stage 9 embryos embedded in plastic resin

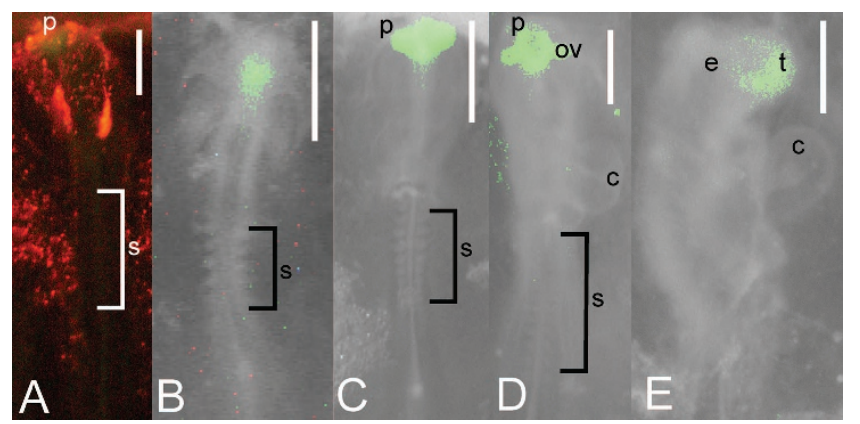

Figure 3. eGFP signals driven by chicken CNE3 at the neurula stage. (A) Anatomical region of successful electroporation visualized using a DsRed signal. (B) HH stage 8 embryo. (C) HH stage 10 embryo. (D) HH stage 12 embryo. (E) HH stage 14 embryo. $p$, prosencephalon; $s$, somites; $c$, cardiac tube; $o v$, optic vesicle; $e$, eye; $t$, telencephalon. Scale bar: $1 \mathrm{~mm}$.
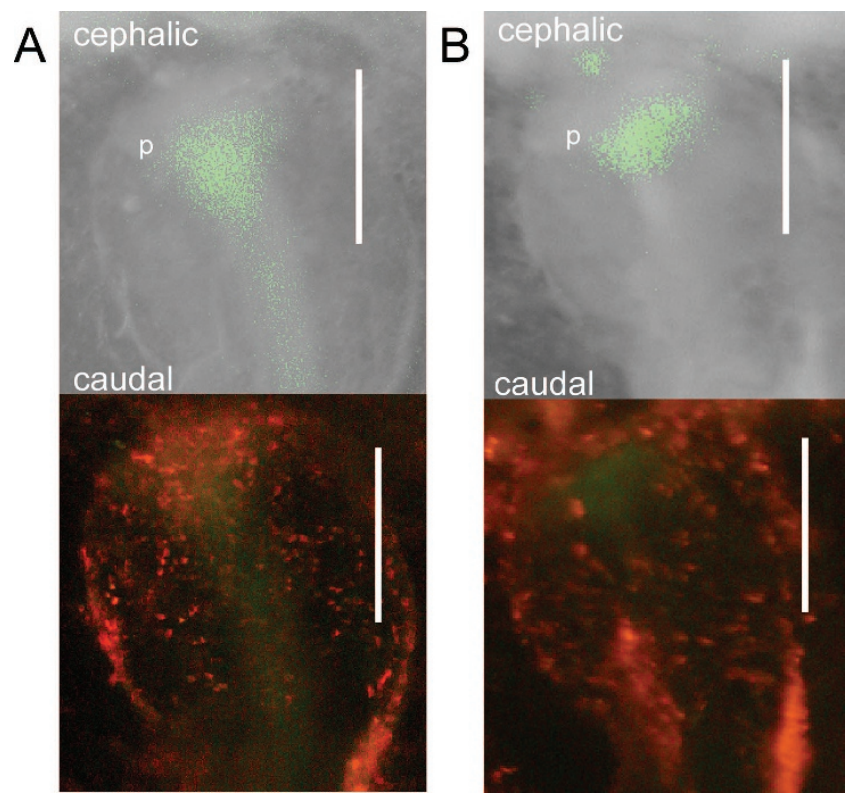

Figure 4. Comparison of enhancer activities of human and chicken CNE3. The upper panels show superimposed images of eGFP and light, bright field images. The lower panels show eGFP/DsRed images. A pair of the upper panel and the lower panel represents the same embryo. (A) HH stage 10 embryo carrying human CNE3. (B) HH stage 10 embryo carrying chicken CNE3. $p$, prosencephalon. Scale bar: $0.5 \mathrm{~mm}$. 

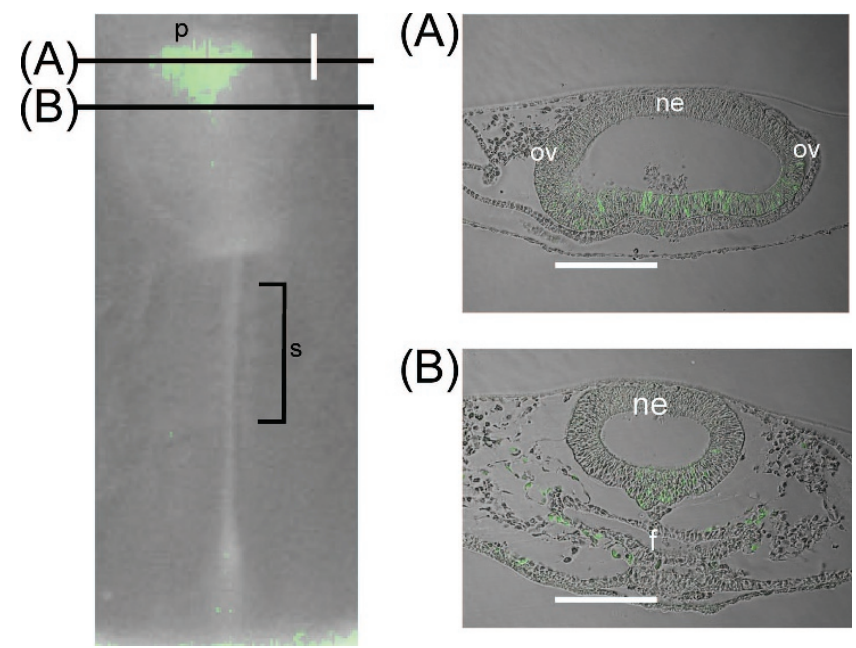

(B)

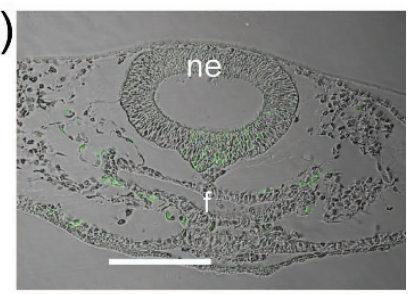

Figure 5. Histologic analysis of eGFP signals driven by chicken CNE3 in $\mathrm{HH}$ stage 9 embryos. (A) Optic vesicle level. (B) Foregut level. The solid lines represent the level of each section. $p$, prosencephalon; $s$, somites; $n e$, neuroepithelium; $o v$, optic vesicle; $f$, foregut. Scale bar: $0.25 \mathrm{~mm}$.
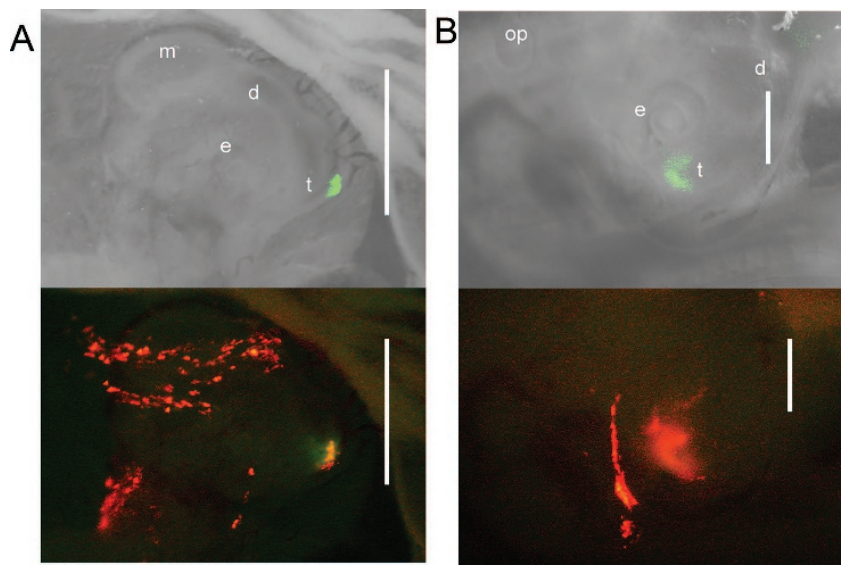

Figure 6. eGFP signals driven by chicken CNE3 at the pharyngula stage. The upper panels show superimposed images of eGFP and light, bright field images. The lower panels show eGFP/DsRed images. A pair of the upper panel and the lower panel represents the same embryo. (A) HH stage 15 embryo. (B) HH stage 20 embryo. $t$, telencephalon; $e$, eye; $d$, diencephalon; $m$, mesencephalon; $o p$, otic placode. Scale bar: $1 \mathrm{~mm}$.

revealed that eGFP signals driven by chicken CNE3 were present predominantly in the ventral neuroepithelium between the levels of the optic vesicles and the foregut (Fig. 5). The maximum signal intensity was observed in the forebrain vesicles at the level of the optic vesicles.

Enhancer activity in pharyngula-stage embryos. The enhancer activity of chicken CNE3 in the later developmental stages of the CNS was investigated after electroporating $\mathrm{HH}$ stage 10 embryos in ovo. The presence of a DsRed signal covering the cephalic region of chick embryos indicated that in ovo electroporation was successful (Fig. 6, $A$ and $B$ ). At the pharyngula stage (HH stage 15), the eGFP signal was confined within the anterior neural ridge, which represents one morphogenetic center known to participate in patterning the anterior neural plate (Fig. 6A) (19). In HH stage 20 embryos, the eGFP signal was detected on the ventral side of the telencephalic vesicle (Fig. $6 B$ ). Serial axial sections of HH stage 15
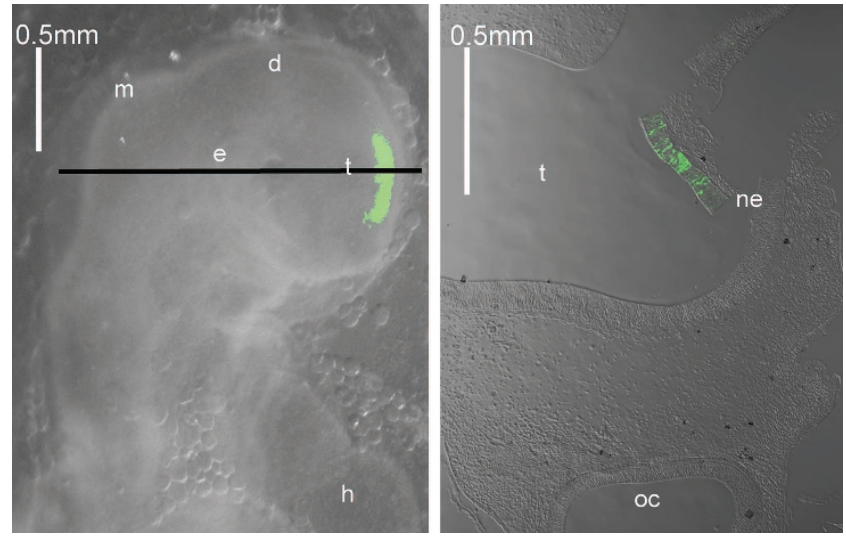

Figure 7. Histologic analysis of eGFP signals driven by chicken CNE3 in $\mathrm{HH}$ stage 15 embryos. The solid line represents the level of the section. $t$, telencephalon; $e$, eye; $d$, diencephalon; $m$, mesencephalon; $h$, heart; $o c$, optic cup; $n e$, neuroepithelium. Scale bar: $0.5 \mathrm{~mm}$.
A

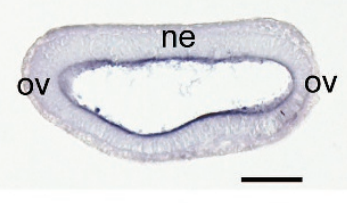

B

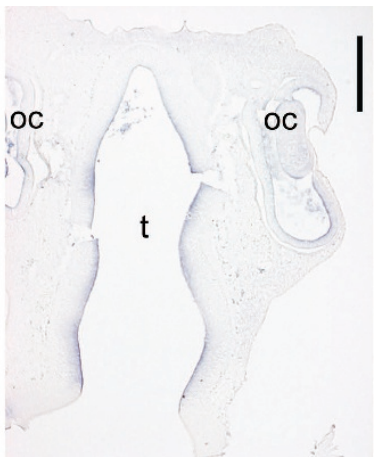

Figure 8. csall in situ hybridization analysis. (A) HH stage 11 embryo. ov, optic vesicle; ne, neuroepithelium. (B) HH stage 20 embryo. $o c$, optic cup; $t$, telencephalon. Scale bar: $0.1 \mathrm{~mm}(A) ; 0.5 \mathrm{~mm}(B)$.

embryos revealed that eGFP signals were present only in the neuroepithelium of the ventral telencephalon (Fig. 7).

In situ hybridization analysis. Whole-mount in situ hybridization studies of chick embryos revealed csall expression in the developing brain, limb buds, and tail bud, a pattern that recapitulates that previously reported by others $(9,10)$. Histologic studies revealed that csall was expressed along the entire neuroepithelium at the level of the optic vesicles at stage 11 (Fig. $8 A)$ and on the neuroepithelium of the telencephalic vesicle at stage 20 (Fig. 8B). Hence, the csall gene expression pattern overlapped that of eGFP reporter expression.

Deletion constructs. Reporter eGFP expression cassettes containing chicken CNE3 with various deleted subregions (I-IV) were constructed and electroporated into embryos at the gastrula stage (HH stage 5). The four deletion constructs lacking $92-157$ bp are depicted in Figure 9A. The length of the deleted segments was $110 \mathrm{bp}$ (subregion IV) in CNE3a, $92 \mathrm{bp}$ (subregion III) in CNE3b, 157 bp (subregion II) in CNE3c, and 100 bp (subregion I) in CNE3d. No eGFP expression was observed with the CNE3a and CNE3b-reporter constructs at the neurula stages, whereas CNE3c and CNE3d-reporter constructs showed comparable expression to the original CNE3 reporter construct (Fig. 9B). To refine the minimal region required for enhancer activity, we next designed constructs CNE3e containing larger deletions than CNE3c and CNE3d. 

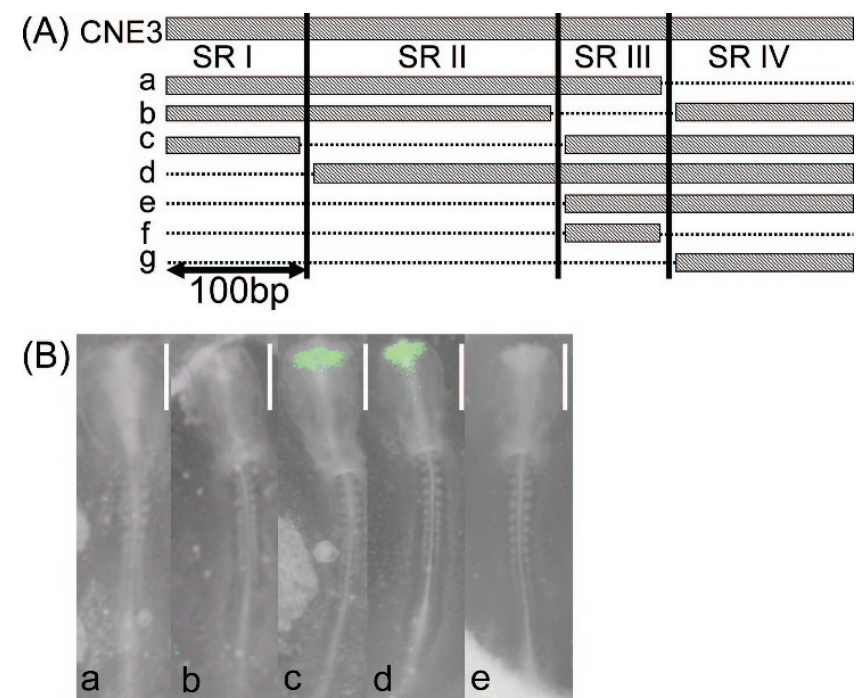

Figure 9. Analysis of chicken CNE3 containing partial deletions. (A) Design of various CNE3 mutants containing partial deletions. The partial deletions are shown by the broken lines. The shaded boxes represent the remaining genomic region of CNE3. The solid lines indicate the boundaries between each subregion $(S R) .(B)$ Enhancer activities of chicken CNE3 containing partial deletions. Scale bar: $1 \mathrm{~mm}$.

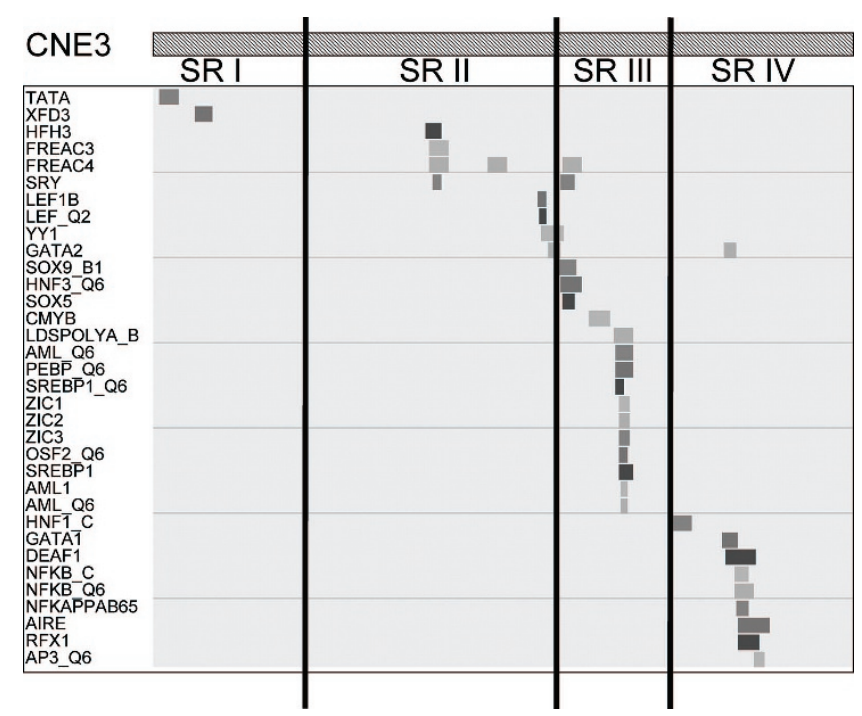

Figure 10. Transcription factor binding sites in CNE3 predicted using $\mathrm{rV}$ ISTA2.0 software. The solid lines indicate the boundaries between each subregion.

Although CNE3e was included in the preserved regions in both CNE3c and CNE3d, CNE3e did not possess any enhancer activity. Further subdivision of CNE3e into CNE3f and CNE3g also eliminated enhancer activity (data not shown).

Prediction of transcription factor binding sites. Transcription factor binding sites found in both the chicken and human CNE3 sequences were predicted using rVISTA2.0 software. The numbers of putative transcription factor binding sites conserved in the chicken and human genomes were 2 in subregion I, 8 in subregion II, 17 in subregion III, and 10 in subregion IV (Fig. 10). One cluster of transcription factor binding sites was found in each of subregions III and IV. Potential binding sites for SOX9, ZIC1, ZIC2, and ZIC3 were clustered in subregion III.

\section{DISCUSSION}

We defined a control element, CNE3, within the SALL1 gene that directs spatio-temporal regulation in the forebrain at the neurula and pharyngula stages. The spatial distribution of the enhancer activity at the neurula stage recapitulated the SALL1 expression pattern previously demonstrated using in situ hybridization (7-10). The CNE3 element exhibited activity in both the sense and antisense directions, thereby fulfilling the requirements of an enhancer element (20). CNE3 is the first $S A L L 1$ enhancer to be identified. To better understand the molecular mechanism regulating $\mathrm{CNE} 3$, we isolated the essential genomic region of CNE3 required to maintain enhancer activity using CNE3 elements containing four types of deletions. The subregion III and IV elements can be regarded as essential elements for the enhancer activity of CNE3 in that the CNE3a and CNE3b elements, which are devoid of subregions IV and III, respectively, did not exhibit the original CNE3 enhancer activity.

A database search for potential transcription factor binding sites using a pattern-matching approach revealed that the ZIC family protein binding motif was present in subregion III, which is essential for CNE3 enhancer function. The ZIC family protein binding motif is shared among three Zic proteins: Zic1, Zic2, and Zic3. Among these three proteins, ZIC2 is known to play an important role in forebrain morphogenesis (21). Hence, ZIC2 represents a candidate protein that may act as an upstream regulator of CNE3. Subregion III of CNE3 included a potential binding site for another transcription factor, SOX9. SOX9 is expressed in the forebrain; therefore, SOX9 might be an upstream molecule of SALL1 CNE3 $(22,23)$. The discussion expanded above relies on a computerbased prediction, and not on actual experiments. An in vitro binding assay is required to test whether ZIC family or SOX9 proteins indeed bind to CNE3, because these putative binding sites are found randomly in genomic DNA at a high frequency. Indeed, a total of three SOX9 binding sites, three ZIC1 binding sites, three ZIC2 binding sites, and five ZIC3 binding sites were present in intron 1 of human SALL1 genomic sequences, and one SOX9 binding site was present in intron 2 of human SALL1 genomic sequences (data not shown).

CNE3 activity was present only in the ventral aspect of the forebrain, indicating that CNE3 activity is under the control of ventralizing signaling in the developing forebrain. Shh may represent one potential upstream signaling molecule. SALL1 is regulated by Shh signaling in the chick limb bud and mouse embryonic kidney. Farrell et al. (10) documented that forced Shh expression using bead implantation into limb buds resulted in the up-regulation of SALL1 in chicken. Hu et al. (24) revealed that Shh deficiency decreased the expression of SALL1 in embryonic kidney using Shh knockout mice. In the developing CNS and paraxial mesoderm of fish (medaka), Koster et al. (25) documented that ectopic Shh activity expanded the Sal expression domain.

The observation that the CNE3e element, which includes subregions III and IV, did not exert enhancer activity indicates that the presence of the CNE3e element is not sufficient for enhancer activity. We have hypothesized that unidentified 
critical transcription factor binding site(s) may be present within subregions I and II, given that the current database of consensus binding sequences for transcription factors remains incomplete.

We successfully identified a forebrain-specific enhancer element using a comparative genomic approach, reconfirming the notion that genome comparisons are helpful for identifying regulatory elements $(1,2)$. We investigated five $\mathrm{CNE}$ and found that one of the five elements conferred enhancer activity. We identified one enhancer out of five CNE. Hence, the sensitivity of our enhancer screening was $20 \%$. This figure is significantly lower than that of other enhancer screening experiments in other species (i.e. 40-90\%) $(3,26)$. The most likely explanation for this seemingly low sensitivity figure is the technical limitation of our assay system being based on a cultured chick embryonic system, since this system does not allow us to observe enhancer activity later than the neurula stages. Four apparently "negative" conserved noncoding elements might act as enhancer elements exclusively at developmental stages later than neurulation. Alternatively, the elements may function as repressors, rather than enhancers.

We have not identified critical enhancers that define expression in the limbs, caudal end, and branchial arches, the primordia of the organs affected in Townes-Brocks syndrome. Because we did not evaluate any CNEs outside of the coding regions (i.e. the $5^{\prime}$ and $3^{\prime}$ ends of the SALL1 coding region), we cannot comment on whether some of these CNE may regulate $S A L L 1$ in the limbs, caudal end, and branchial arches.

In the present study, we have illustrated that a relatively simple screen using the principle of comparative genomics and electroporation into chick embryos can identify tissuespecific enhancers. From a basic science research standpoint, systematic discovery of tissue-specific enhancer elements would advance our understanding on complex network of developmental regulatory genes. From a translational research standpoint, identification of tissue-specific enhancer elements will facilitate designing safer gene transfer strategies that will minimize adverse events.

Acknowledgments. The authors thank Hitoshi Abe and Yuko Hashimoto for technical support, and Kyoko Shinohara for secretarial assistance.

\section{REFERENCES}

1. Uchikawa M, Takemoto T, Kamachi Y, Kondoh H 2004 Efficient identification of regulatory sequences in the chicken genome by a powerful combination of embryo electroporation and genome comparison. Mech Dev 121:1145-1158
2. Bejerano G, Pheasant M, Makunin I, Stephen S, Kent WJ, Mattick JS, Haussler D 2004 Ultraconserved elements in the human genome. Science 304:1321-1325

3. Woolfe A, Goodson M, Goode DK, Snell P, McEwen GK, Vavouri T, Smith SF, North P, Callaway H, Kelly K, Walter K, Abnizova I, Gilks W, Edwards YJ, Cooke JE, Elgar G 2005 Highly conserved non-coding sequences are associated with vertebrate development. PLoS Biol 3:e7

4. Kohlhase J, Schuh R, Dowe G, Kuhnlein RP, Jackle H, Schroeder B, SchulzSchaeffer W, Kretzschmar HA, Kohler A, Muller U, Raab-Vetter M, Burkhardt E, Engel W, Stick R 1996 Isolation, characterization, and organ-specific expression of two novel human zinc finger genes related to the Drosophila gene spalt. Genomics 38:291-298

5. Townes PL, Brocks ER 1972 Hereditary syndrome of imperforate anus with hand, foot, and ear anomalies. J Pediatr 81:321-326

6. Kohlhase J, Wischermann A, Reichenbach H, Froster U, Engel W 1998 Mutations in the SALL1 putative transcription factor gene cause Townes-Brocks syndrome. Nat Genet 18:81-83

7. Buck A, Kispert A, Kohlhase J 2001 Embryonic expression of the murine homologue of SALL1, the gene mutated in Townes-Brocks syndrome. Mech Dev 104:143-146

8. Nishinakamura R, Matsumoto Y, Nakao K, Nakamura K, Sato A, Copeland NG Gilbert DJ, Jenkins NA, Scully S, Lacey DL, Katsuki M, Asashima M, Yokota T 2001 Murine homolog of SALL1 is essential for ureteric bud invasion in kidney development. Development 128:3105-3115

9. Sweetman D, Smith TG, Farrell ER, Munsterberg A 2005 Expression of csall in pre limb-bud chick embryos. Int J Dev Biol 49:427-430

10. Farrell ER, Munsterberg AE 2000 csall is controlled by a combination of FGF and Wnt signals in developing limb buds. Dev Biol 225:447-458

11. Uchikawa M, Ishida Y, Takemoto T, Kamachi Y, Kondoh H 2003 Functional analysis of chicken Sox2 enhancers highlights an array of diverse regulatory elements that are conserved in mammals. Dev Cell 4:509-519

12. Ovcharenko I, Loots GG, Giardine BM, Hou M, Ma J, Hardison RC, Stubbs L, Miller W 2005 Mulan: multiple-sequence local alignment and visualization for studying function and evolution. Genome Res 15:184-194

13. Loots GG, Ovcharenko I 2004 rVISTA 2.0: evolutionary analysis of transcription factor binding sites. Nucleic Acids Res 32:W217-W221

14. Ho SN, Hunt HD, Horton RM, Pullen JK, Pease LR 1989 Site-directed mutagenesis by overlap extension using the polymerase chain reaction. Gene 77:51-59

15. Hamburger V, Hamilton HL 1951 A series of normal stages in the development of the chick embryo. J Morphol 88:49-92

16. Muramatsu T, Mizutani Y, Ohmori Y, Okumura J 1997 Comparison of three nonviral transfection methods for foreign gene expression in early chicken embryos in ovo. Biochem Biophys Res Commun 230:376-380

17. Aramaki M, Kimura T, Udaka T, Kosaki R, Mitsuhashi T, Okada Y, Takahashi T, Kosaki K 2007 Embryonic expression profile of chicken CHD7, the ortholog of the causative gene for CHARGE syndrome. Birth Defects Res A Clin Mol Teratol 79:50-57

18. Boardman PE, Sanz-Ezquerro J, Overton IM, Burt DW, Bosch E, Fong WT, Tickle C, Brown WR, Wilson SA, Hubbard SJ 2002 A comprehensive collection of chicken cDNAs. Curr Biol 12:1965-1969

19. Rubenstein JL, Beachy PA 1998 Patterning of the embryonic forebrain. Curr Opin Neurobiol 8:18-26

20. Blackwood EM, Kadonaga JT 1998 Going the distance: a current view of enhancer action. Science 281:60-63

21. Brown LY, Kottmann AH, Brown S 2003 Immunolocalization of Zic2 expression in the developing mouse forebrain. Gene Expr Patterns 3:361-367

22. Pompolo S, Harley VR 2001 Localisation of the SRY-related HMG box protein, SOX9, in rodent brain. Brain Res 906:143-148

23. Wright E, Hargrave MR, Christiansen J, Cooper L, Kun J, Evans T, Gangadharan U, Greenfield A, Koopman P 1995 The Sry-related gene Sox9 is expressed during chondrogenesis in mouse embryos. Nat Genet 9:15-20

24. Hu MC, Mo R, Bhella S, Wilson CW, Chuang PT, Hui CC, Rosenblum ND 2006 GLI3-dependent transcriptional repression of Gli1, Gli2 and kidney patterning genes disrupts renal morphogenesis. Development 133:569-578

25. Koster R, Stick R, Loosli F, Wittbrodt J 1997 Medaka spalt acts as a target gene of hedgehog signaling. Development 124:3147-3156

26. Pennacchio LA, Ahituv N, Moses AM, Prabhakar S, Nobrega MA, Shoukry M, Minovitsky S, Dubchak I, Holt A, Lewis KD, Plajzer-Frick I, Akiyama J, De Val S, Afzal V, Black BL, Couronne O, Eisen MB, Visel A, Rubin EM 2006 In vivo enhancer analysis of human conserved non-coding sequences. Nature 444:499_ 502 